\title{
Designing Cooperative IS: Exploring and Evaluating Alternatives
}

\author{
Volha Bryl, Paolo Giorgini, and John Mylopoulos \\ Department of Information and Communication Technology University of Trento, \\ via Sommarive 14, 38050 Povo (TN), Italy, \\ $\{$ bryl, paolo.giorgini, jm\} @dit.unitn.it
}

\begin{abstract}
At the early stages of the cooperative information system development one of the major problems is to explore the space of alternative ways of assignment and delegations of goals among system actors. The exploration process should be guided by a number of criteria to determine whether the adopted alternative is good-enough. This paper frames the problem of designing actor dependency networks as a multi-agent planning problem and adopts an off-the-shelf planner to offer a tool (P-Tool) that generates alternative actor dependency networks, and evaluates them in terms of metrics derived from Game Theory literature. As well, we offer preliminary experimental results on the scalability of the approach.
\end{abstract}

\section{Introduction}

During the requirements analysis and design of cooperative information systems one has to cope with the fundamental problem of planning, i.e. finding optimal/good-enough delegations to a set of system actors which collectively fulfill a given set of goals. These goals are initially assigned to the actors who may not have enough capabilities to satisfy them, so they are decomposed and delegated to other actors, thereby creating networks of delegations. The process ends when all initial goals can be fulfilled if all system actors deliver on their delegations.

Exploring the space of alternative dependency networks is a difficult design task. This is so because such networks represent complex socio-technical systems where organizational, human and system actors depend on each other to fulfill root-level goals. Moreover, there are no generic criteria to guide the design process by determining whether a solution is good-enough, or even optimal. Our ultimate goal is to identify suitable metrics for evaluating alternative sets of delegations to help a designer select the best one.

The purpose of this paper is to propose a framework for the automatic selection and evaluation of alternative dependency networks, or design alternatives. The framework supports both the generation and evaluation of alternatives. Specifically, the framework adopts multi-agent planning techniques and uses an off-the-shelf planning tool. Alternatives are evaluated with respect to individual interests of system actors (i.e. their own goals). Ideas from Game Theory [14] are used to determine whether an alternative is an equilibrium. In particular, an alternative is an equilibrium if no actor can do better 
with respect to its own goals by adopting a different strategy for delegating and accepting delegations. When combined together, these two steps support the designer of an information system in selecting alternatives that are in equilibrium with respect to the local strategies of each actor. An early version of this idea is used in [3] to propose a framework to generate alternative designs for secure systems. This paper goes further by describing a prototype tool that generates alternatives, presents some experimental results, and also proposes the evaluation techniques for alternatives based on game-theoretic notions.

The process of the best alternative selection consists of the following steps:

1. Identify system and human actors, goals and their properties. Define goal decompositions and dependency relationships among actors.

2. For each actor identify criteria to evaluate alternatives.

3. Automatically explore the space of alternatives "on the upper level" to identify assignments of coarse-grained goals to actors.

4. Separately for each actor, automatically explore the alternative ways to satisfy the goals the actor was assigned at step 3. According to above identified evaluation criteria, select "the best" alternative for each actor. During this step, alternative refinements of coarse-grained goals and delegation dependencies among actors are explored.

5. Evaluate the combined solution consisting of alternatives identified at step 4. In case it does not satisfy one or several system actors (e.g. they are overloaded with respect to others), return to step 4 to search for another alternative.

Ideally, the process stops after a number of iterations when the socio-technical structure is optimized enough to comply with the individual interests of the system actors. If no satisfactory alternatives can be generated at some step, the designer should return to steps 1 or 2, and revise either the initial structure, or the evaluation criteria.

Figure 1a presents a simple example of the problem of exploring design alternatives. Note that in this paper we use the $i^{*}$ graphical notation [20]. The $i^{*}$ modeling framework and an associated requirements analysis process (Tropos [2]) are based on the intentional concepts of an actor, a goal and a social dependency, and supports modeling and analysis during the early requirements engineering phase. So, in the example Actor 1 has to achieve a Goal, which can be refined into two subgoals Subgoal 1 and Subgoal 2. The actor can decide to achieve the goal by itself or delegate it to Actor 2. In both cases, there are a number of alternative ways that can be adopted. So, for instance, Actor 1 can decide to delegate to Actor 2 the whole Goal (Figure 1b), or a part of it (Figure 1c). Shaded goal in the circle of an actor means that the goal is the responsibility of this actor. Even for this primitive example, exploring all the alternatives is quite tedious, and a support for alternative generation and evaluation would be beneficial.

The rest of the paper is structured as follows. In the next section we introduce the example we use through the paper to describe our framework. In Section 3 the issue of alternative generation and evaluation is detailed. Section 4 describes the P-Tool, an implemented prototype tool to support the exploration of alternatives, and reports some experimental results. Finally, in Section 5 we describe the related work, and discuss conclusions and future work directions in Section 6. 


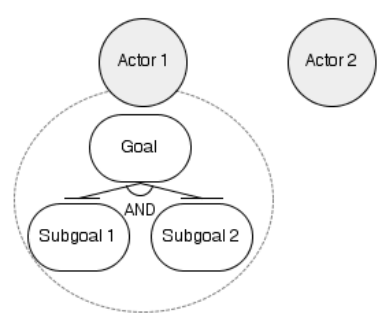

(a) Sample problem

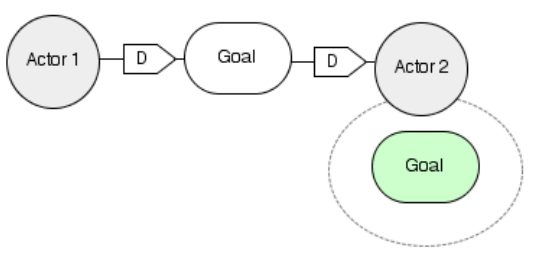

(b) 1 st alternative

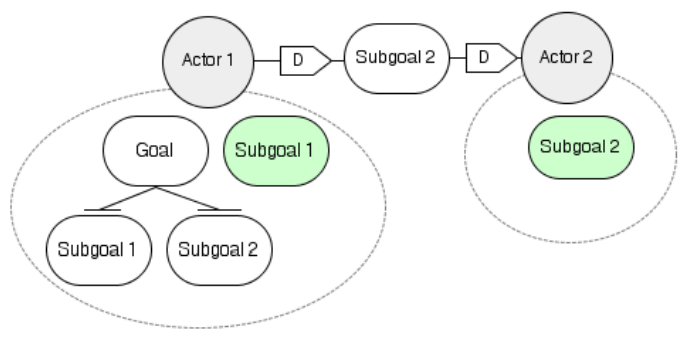

(c) 2nd alternative

Fig. 1. Sample problem and two alternative solutions

\section{SDS System Example}

Let us consider a small software development company, which typical projects are medium-scale web based information systems (like, e.g. online library catalog, or travel agency home page with online trip booking, etc.). Within the company there are three teams of developers each focused on its area: GUI development, web design, and database support. Each team can develop subcomponents and/or consult other teams on questions related to their expertise. A manager is supposed to divide the project into meaningful parts and perform the assignment of goals to achieve to the development teams. Note, that a manager can assess how the project goals are refined and what skills are required to satisfy each subgoal only on the coarse-grained level.

The company has decided to use a software development support system (SDS system or supporting system in the following), which will facilitate and report communication among actors, archive a library of reusable components, organize the search for such components, store and provide the information specific to the project under 


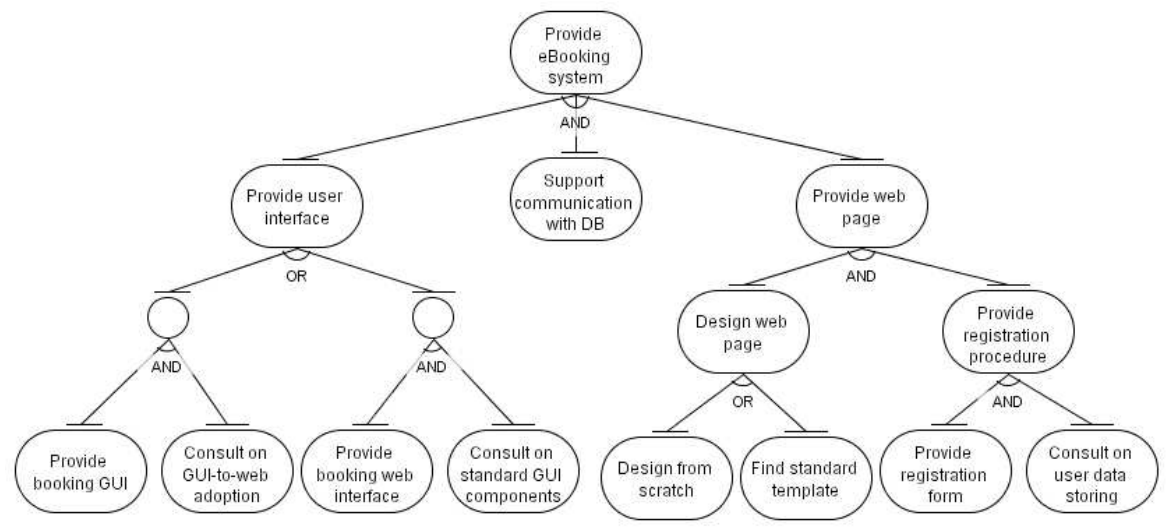

Fig. 2. Goal tree for eBooking project

development (e.g. contain a glossary of domain specific terms, store domain specific classifications, etc.). Communication between manager and members of the development teams is supposed to be carried out only through the supporting system. Teams can communicate with each other in two cases: when one team wants to redirect a subgoal which requires the development skills these team does not possess to another team, and when one team needs to consult another one. The first type of communication is possible only through the supporting system, while the communication on consultancy can be done both through the supporting system and e-mail (or even personal communication).

To analyze the above described socio-technical system let us consider a typical project it might deal with: web based eBooking system for the travelling agency. As it is represented in Figure 2, the high-level goal provide eBooking system is refined into three subgoals: provide user interface, support communication with $D B$ and provide web page. In order to fulfil the high-level goal, all three subgoals should be satisfied. Two subgoals are further refined, e.g. provide eBooking system subgoal can be reached in two alternative ways: by developing eBooking GUI and consulting web designers to adopt it for web environment, or by developing web booking interface together with consulting GUI team on which standard components to use.

The OR-decomposition of the subgoals provide user interface and design web page introduces alternative solutions for the development of the eBooking system. One of the alternatives to achieve provide user interface subgoal is depicted in Figure 3. The goal is decomposed by GUI team actor, which selects the leftmost alternative among the two or-subgoals. The selected subgoal is further decomposed into two subgoals: consult on standard GUI components and provide booking web interface. The former is satisfied by GUI team, while the latter is delegated to Web design team. 


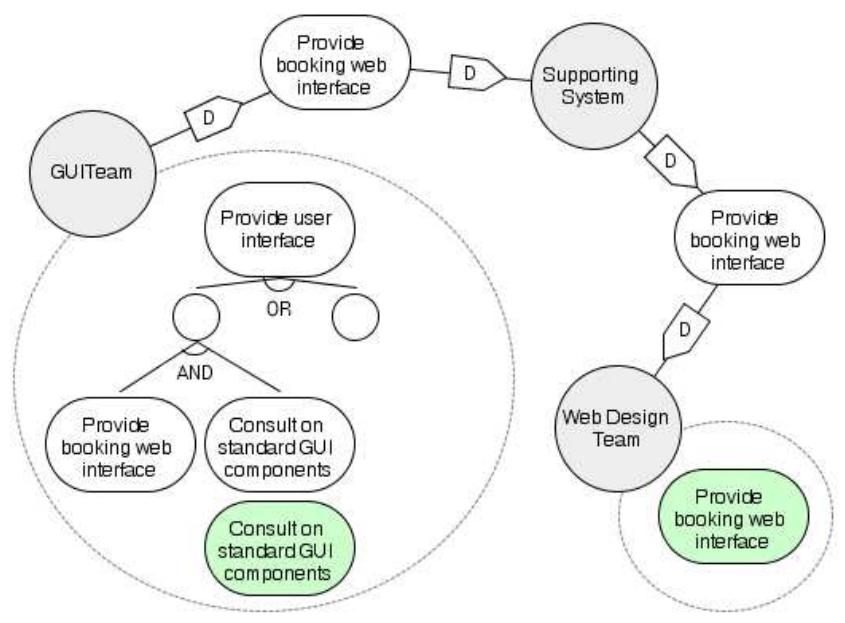

Fig. 3. An alternative way to achieve provide user interface subgoal

\section{Exploring and Evaluating Alternatives}

\subsection{Formalization of the Planning Problem}

As it was discussed in the Introduction, requirements to the information systems are conceived as networks of delegations among actors - at least within the frameworks such as $i^{*}$ [20], Tropos [2] and the like. Every delegation involves two actors, where one actor delegates to the other the fulfillment of a goal. The delegatee can either fulfill delegated goal, or further delegate it, thus creating another delegation relation in the network. Intuitively, these can be seen as actions that the designer/requirements engineer ascribes to the members of the organization and the system-to-be. Further, the task of constructing such networks can be framed as a planning problem: selecting a suitable requirements structure corresponds to selecting a plan that satisfies the goals of human and software agents.

Thus, we have chosen the AI planning approach to support the designer/requirements engineer in the process of selecting the best design alternative. The basic idea behind planning approach is to automatically determine the course of actions (i.e. a plan) needed to achieve a certain goal where an action is a transition rule from one state of the system to another $[19,15]$. Actions are described in terms of preconditions and effects: if the precondition is true in the current state of the system, then the action is performed. As a consequence of an action, the system will be in a new state where the effect of the action is true.

Planning approach requires a specification language to represent the planning domain, i.e.

- the initial state of the system;

- the goal of the planning problem (i.e. the desired final state of the system);

- the description of actions; 
- the axioms of background theory.

Once the domain is described, the solution to the planning problem is the (not necessarily optimal) sequence of actions that allows the system to reach the desired state from the initial state.

To describe the initial state of the system we should specify actors' and goal properties, and social relations among actors. We propose to represent initial state in terms of predicates that correspond to

- the possible ways of goal decomposition;

- actors' capabilities and desires to achieve a goal;

- possible delegation relations among actors.

The desired state of the system (or the goal of the planning problem) is described through the conjunction of predicates derived from the description of actors' desires in the initial state. Essentially, for each desired goal a predicate is added to the goal of the planning problem.

Different types of logic could be applied for this purpose, e.g. first order logic is often used to describe the planning domain with conjunctions of literals specifying the states of the system. In Table 1 predicates used to describe the domain of information system design are introduced. Predicates take variables of three types: actors, goals and goal types. To typify goals, type predicate is used. Actor capabilities are described with can_decompose and can_decompose_gt predicates, which means that an actor has enough capabilities to satisfy a specific goal or any goal of a specific type, accordingly. Social dependencies among actors are reflected by can_depend_on and can_depend_on_gt predicates, which means that one actor can delegate to another actor the fulfilment of any goal or, in the latter case, any goal of a specific type. Predefined ways of goal refinement are represented using decomposition predicates, while with can_decompose_gt the scope of each actor can be represented: an actor can refine, or knows how to refine, only goals within his scope. Initial actors' desires are represented with wants predicate. When the goal is fulfilled satisfied predicated becomes true for it.

In Figure 4, a part of SDS System example formalization is presented. The goal types $t$ Consult, $t$ WDDevel and ManagScope are used.

In $i * /$ Tropos approach, when drawing the model of a system, the designer/requirements engineer assigns goals to actors, defines delegations of goals from one actor to another, and identifies appropriate goal refinements among the predefined alternative refinements. Thus, the following actions will be used by a planner to find a way to fulfill the goals of the system actors.

Goal satisfaction. An actor can satisfy a goal only if the achievement of the goal is among his desires and he can actually satisfy it. The effect of this action is the fulfillment of the goal.

Goal delegation. An actor may have not enough capabilities to achieve his goals by himself, and so he has to delegate their satisfaction to other actors. This passage of responsibilities is performed only if the delegator wants a goal to be achieved and can depend on the delegatee to achieve it. The effect of this action is that the delegator does not worry any more about the satisfaction of the goal, while the delegatee takes the responsibility for the fulfillment of the goal and so it becomes his 


\begin{tabular}{|c|}
\hline Goal Properties \\
\hline $\begin{array}{l}\text { type(g: goal, gt : gtype) } \\
\text { subtype(child : gtype, parent : gtype }) \\
\text { and_decomposition }_{\mathrm{n}}\left(\mathrm{g}: \text { goal, } \mathrm{g}_{1}: \text { goal }, \ldots, \mathrm{g}_{\mathrm{n}}: \text { goal }\right) \\
\text { or_decomposition }_{\mathrm{n}}\left(\mathrm{g}: \text { goal, } \mathrm{g}_{1}: \text { goal, } \ldots, \mathrm{g}_{\mathrm{n}}: \text { goal }\right) \\
\text { satisfied }(\mathrm{g}: \text { goal })\end{array}$ \\
\hline Actor Properties \\
\hline $\begin{array}{l}\text { can_satisfy(a : actor, g : goal) } \\
\text { can_satisfy_gt(a : actor, gt : gtype }) \\
\text { can_decompose_gt }(a: \text { actor, gt : gtype }) \\
\text { wants(a : actor,g : goal })\end{array}$ \\
\hline Actor Relations \\
\hline $\begin{array}{l}\text { can_depend_on(a : actor, b : actor) } \\
\text { can_depend_on_gt (a : actor, b : actor, gt : gtype) }\end{array}$ \\
\hline
\end{tabular}

Table 1. Primitive predicates

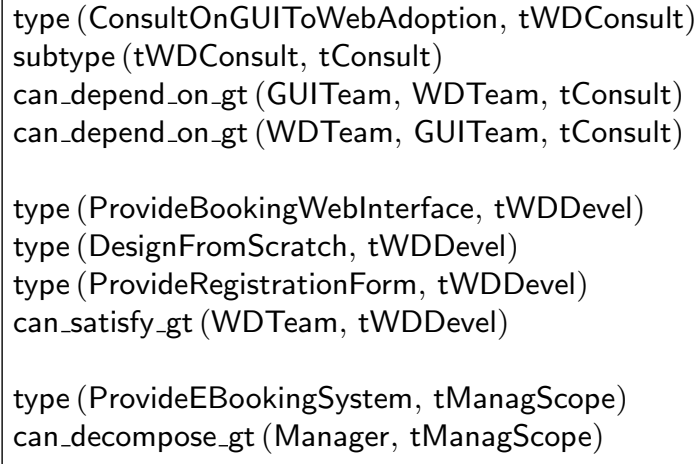

Fig. 4. Predicates for SDS System example

own desire to achieve it. The delegator does not care how the delegatee satisfies the goal (e.g. by his own capabilities or by further delegation), it is up to the delegatee to decide it.

Goal decomposition/refinement. As in different goal-oriented modeling frameworks (e.g. as in Tropos and KAOS [5]) two types of goal refinement are supported: ORdecomposition, which suggests the list of alternative ways to satisfy the goal, and AND-decomposition, which refines the goals into subgoals which all are to be satisfied in order to satisfy the initial goal. An actor can decompose a goal only if he wants it to be satisfied, and only in the way which is predefined in the initial state of the system. The effect of decomposition is that the actor who refines the goal focuses on the fulfillment of subgoals instead of the initial goal. It is assumed that different actors can decompose the same goal in different ways.

In addition to actions, axioms of the planning domain can be defined. These are rules that hold in every state of the system and are used to complete the description of 
the current state. For example, to propagate goal properties along goal refinement the following axiom is used: a goal is satisfied if all its and-subgoals or at least one of the or-subgoals are satisfied.

\subsection{Evaluation Procedure}

The alternative designs generated by the planner should be evaluated, amended and approved by the designer. The tricky point here is the solution evaluation which can be complex enough even for experienced designers with considerable domain expertise. Alternative requirements structures can be evaluated both from global and local perspectives, i.e. from the designer's point of view and from the point of view of individual actors. The optimality of a solution in the global sense could be assessed with respect to the following.

- Length of the obtained plan. The number of actions in the obtained plan is often the criteria for the planner itself to prefer one solution to another. Thus, it can be assumed that the obtained plan is already (locally) optimal in the sense of the length minimization.

- Overall plan cost. This is closely related with the idea of plan metrics introduced in PDDL 2.1 [8]. Plan metrics specify the basis on which a plan is evaluated for a particular problem (e.g. action costs or duration), and are usually numerical expressions to be minimized or maximized. However, the complexity of the problem of optimizing a solution with respect to the defined metrics is very high and the feature is still poorly supported by the available planning tools [8].

- Degree of satisfaction of non-functional requirements. E.g. in [12], a set of rules is proposed to identify application-specific parameters and functions to quantify impacts of different explored alternatives on non-functional goals (e.g. security, performance, usability) satisfaction.

In this paper we are not dealing with the global evaluation of generated alternatives. However, the first point, (sub)optimality of the solution with respect to the plan length, is automatically taken into account by the planner.

Local evaluation of the obtained plan is a much more complex task. Indeed, a challenging characteristic of modern information system design is that human agents should be taken into account. They can be seen as players in a game theoretic sense as they are self-interested and rational. This means they want to minimize the load imposed personally on them, e.g. they want to constraint the number and the complexity of actions they are involved in ${ }^{1}$. In a certain sense non-human agents, i.e. system components, are players as well as it is undesirable to overload them. Each player has a set of strategies he could choose from, e.g. he could decide whether to satisfy a goal himself or to pass it further to another system actor. Strategies are based on the player's capabilities and his relations (e.g. subordination, friendship, or trust - all represented as possible dependencies in our framework) with other human and artificial agents in the system.

\footnotetext{
${ }^{1}$ In this work we focus on the load constraints only, and do not consider other factors which influence the player's decision to deviate, e.g. risk concerns.
} 


\begin{tabular}{|c|l|l|}
\hline Action & Cost & \multicolumn{1}{|c|}{ Actors and Goals } \\
\hline Satisfy & 3 & $\begin{array}{l}\text { goals of type } t \text { Consult for WDTeam, GUITeam and DBTeam; } \\
\text { goal FindStandardTemplate for SupportingSystem }\end{array}$ \\
\hline & 4 & $\begin{array}{l}\text { goals of type } t \text { WDDevel for WDTeam, goal ProvideBookingGUI } \\
\text { for GUITeam; goal SupprtDBCommunication for DBTeam }\end{array}$ \\
\hline Delegate & 1 & $\begin{array}{l}\text { SupportingSystem; delegations between WDTeam, GUITeam } \\
\text { and DBTeam }\end{array}$ \\
\hline & 2 & all other actors and goals \\
\hline Refine & 2 & all actors and goals \\
\hline
\end{tabular}

Table 2. Costs for the SDS System example

The substantial difficulty in applying game theoretic ideas to our problem is that all actors of an information system should work cooperatively as a solid mechanism satisfying the overall organizational goal. Differently from classical non-cooperative game theory, where all players choose their strategies independently and simultaneously before the game, in our problem actors' choices are closely interrelated. A player cannot independently change his strategy because the new action sequence will very likely be unsatisfactory, i.e. it will not be a solution anymore. Thus, to satisfy the system goals it will be necessary to impose some additional load (to compensate the load this player tries to avoid) on some other actors - and it might happen that they will not be satisfied with their new utilities, and will try to deviate from the strategy they were imposed, and so on and so forth. Thus, if one actor wants to deviate from the generated solution, the re-planning is needed to search for another alternative option, which is then evaluated, possibly, to be re-plan again. The process stops when a (sub)optimal design alternative option is found. In our framework the following "replan-towards-optimality" procedure is used.

First, for all actors $a_{i}, i=\overline{1, n}$ and all goals $g_{k}, k=\overline{1, m}$, where $n$ and $m$ are the number of actors and goals, respectively, the costs are defined:

- $c s_{i k}$ is the cost for the actor $a_{i}$ of satisfying the goal $g_{k}$;

- $c r_{i k}$ is the cost for the actor $a_{i}$ of refining the goal $g_{k}$;

- $c d_{i j k}$ is the cost for the actor $a_{i}$ of delegating a goal $g_{k}$ to the actor $b_{j}$.

In Table 2 the costs of actions for actors from the SDS System example are defined.

Then, the cost of a given alternative $P$ for the actor $a_{i}$ is calculated by summing up the costs of actions in $P$ which $a_{i}$ is involved in, and is denoted by

$$
\begin{gathered}
c\left(P, a_{i}\right)=\sum_{\text {delegate }\left(a_{i}, b_{j}, g_{k}\right) \in P} c d_{i j k}+ \\
\sum_{\text {decompose }_{l}\left(a_{i}, g_{k}, g_{k 1}, \ldots, g_{k l}\right) \in P} c r_{i k}+\sum_{\text {satisfy }\left(a_{i}, g_{k}\right) \in P} c s_{i k},
\end{gathered}
$$

where decompose $e_{l}\left(a_{i}, g_{k}, g_{k 1}, \ldots, g_{k l}\right)$ stands for the decomposition of $g_{k}$ into $l$ subgoals $g_{k 1}, \ldots, g_{k l}$.

If $P$ is the alternative depicted in Figure 3 , then $c(P$, GUITeam $)=6$, $c(P, W D T e a m)=6$ and $c(P$, SupportingSystem $)=2$. 
Note, that in our framework we do not use the notion of utility, which is an important game theory construct. This is done mainly for the simplicity reasons. The utility of an alternative $P$ for the actor $a_{i}$ can be defined as the difference between maximum upper bound for the solution cost for actor $a_{i}$ and $c\left(P, a_{i}\right)$. Basically, utility says how much an actor "saves" with the alternative $P$ being selected.

After the costs are computed, for each actor the conditions are defined upon which an actor decides whether to deviate from an alternative $P$ or not. The conditions could be either one of the following, or both.

- Actor $a_{i}$ whose predefined upper cost bound $c_{i}^{u p}$ is greater than $c\left(P, a_{i}\right)$ is willing to deviate from $\mathrm{P}$.

- Actor $a_{i}$ whose predefined upper bound $c d e v_{i}^{u p}$ on cost deviation is greater than $c\left(P, a_{i}\right)-\operatorname{avg}_{i}\left(c\left(P, a_{i}\right)\right)$ wants to deviate from $P$.

In this work we consider only the first deviation condition - predefined upper cost bounds.

Finally, the evaluation procedure is the following.

- An alternative $P$ is generated with the help of planner.

- Cost $c\left(P, a_{i}\right)$ is calculated for each $a_{i}$.

- Actor $a_{\min }$ is identified whose value of $c(.,$.$) is minimal among all actors who$ want to deviate from $P$.

- The first most expensive action $d_{\text {worst }}$ (the one with the highest cost) is identified among actions of $P$ in which $a_{m i n}$ is involved.

- Negation of $d_{\text {worst }}$ is added to the initial planning problem, and replanning is performed. If no plan can be found, the next $d_{\text {worst }}$ is identified.

The process stops when an equilibruium-like solution is found, i.e. no actors are willing to deviate from it and the designer approves this solution. The designer remains in the process all the time, and can stop the iterations whenever he thinks the satisficing alternative is generated.

This evaluation procedure is used at the following steps of the selection of the best alternative, defined in the Introduction.

- At step 3, while selecting the best assignments of coarse-grained goals to actors.

- At step 4, separately for each actor, when exploring the ways to satisfy the goals the actor was assigned.

- At step 5, when evaluating the combined solution consisting of alternatives identified at step 4 . Here the replanning is performed only for the alternative to which $d_{\text {worst }}$ belongs to.

\section{P-Tool and Experiments}

\subsection{Choosing the Planner}

One important step we have performed during the implementation of the proposed framework, is choosing the "right planner" among off-the-shelf tools available. In the last years many planners have been proposed [15]. In order to choose one of them the following requirements are considered: 


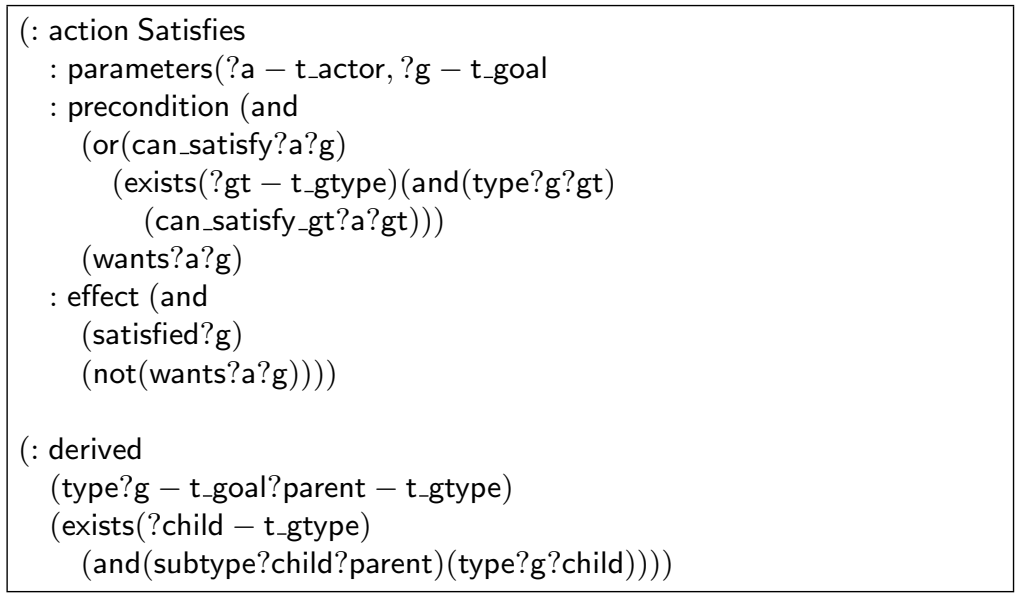

Fig. 5. Domain description using PDDL

- The planner should not produce redundant plans. Under non-redundant plan we mean that, by deleting an arbitrary action of the plan, the resulting plan is no more a "valid" plan (i.e. it does not allow to reach the desired state from the initial state).

- The planner should use PDDL (Planning Domain Definition Language) since it is becoming a "standard" planning language and many research groups work on its implementation.

- The language should support a number of "advanced" features (e.g. derived predicates) that are essential for implementing our planning domain, i.e. it should be at least PDDL 2.2. [6].

The first requirement is related to the question of the optimality of the generated design decisions. We argue that it is not necessary to focus on the optimal design: human designers do not prove that their design is optimal, why should a system do it? Instead, in our framework the plan is required to be non-redundant, which guarantees at least the absence of alternative delegation paths since a plan does not contain any redundant actions.

We have compared a number of planners with respect to above requirements (see [3] for the details). Finally, we have chosen LPG-td [13], a fully automated system for solving planning problems, supporting PDDL 2.2 specification for implementing our planning domain.

Then, we have implemented our planning domain in PDDL 2.2. Figure 5 presents the specification of one action and one domain axiom in PDDL 2.2.

Figure 6 shows the plans generated by LPG-td for satisfying provide user interface and provide web page subgoals. The former plan is illustrated in Figure 3, the latter in Figure 7.

Preliminary experiments were conducted to test the scalability of the approach. A very simple "core" problem was considered, with three actors $A, B$ and $C$ and two goals, $G_{1}$ and $G_{2}$, which $A$ wants to be achieved, and $B$ and $C$ can satisfy. Then "additional" actors with the dependencies among them were added to the problem, but 


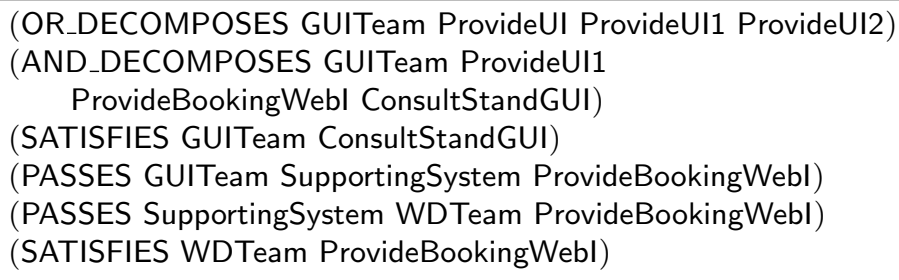

(a) Provide user interface

\begin{tabular}{|l|}
\hline (AND_DECOMPOSES WDTeam ProvideWebPage \\
DesignWebPage ProvideRegistrProc) \\
(AND_DECOMPOSES WDTeam ProvideRegistrProc \\
ProvideRegForm ConsultOnStoreUData) \\
(SATISFIES WDTeam ProvideRegForm) \\
(OR_DECOMPOSES WDTeam DesignWebPage \\
DesignFromScratch FindStandardTemplate) \\
(SATISFIES WDTeam DesignFromScratch) \\
(PASSES WDTeam DBTeam ConsultOnStoreUData) \\
(SATISFIES DBTeam ConsultOnStoreUData)
\end{tabular}

(b) Provide web page

Fig. 6. Plans for ProvideUI and ProvideWebPage subgoals

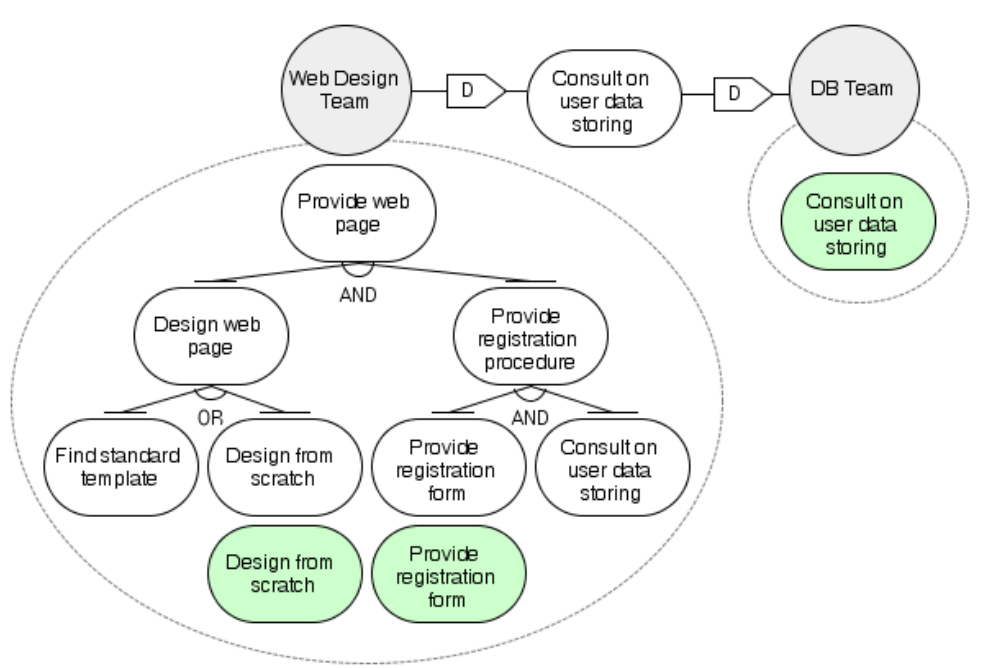

Fig. 7. Diagram for the plan for ProvideWebPage subgoal

they did not interfere at all with "core" subproblem. The idea was to check whether the search time of the plan to achieve $G_{1}$ and $G_{2}$ depends on the number of "additional" actors and dependencies among them. The experiments showed that, at least with respect to this example, the approach is scalable. Basically, the search time for the problem 


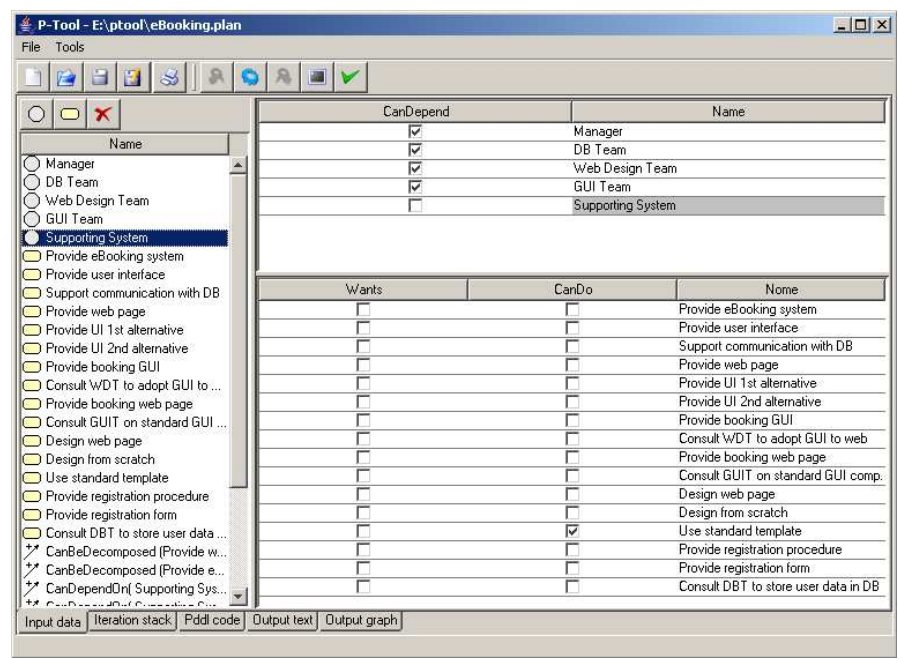

(a) Identifying actor properties

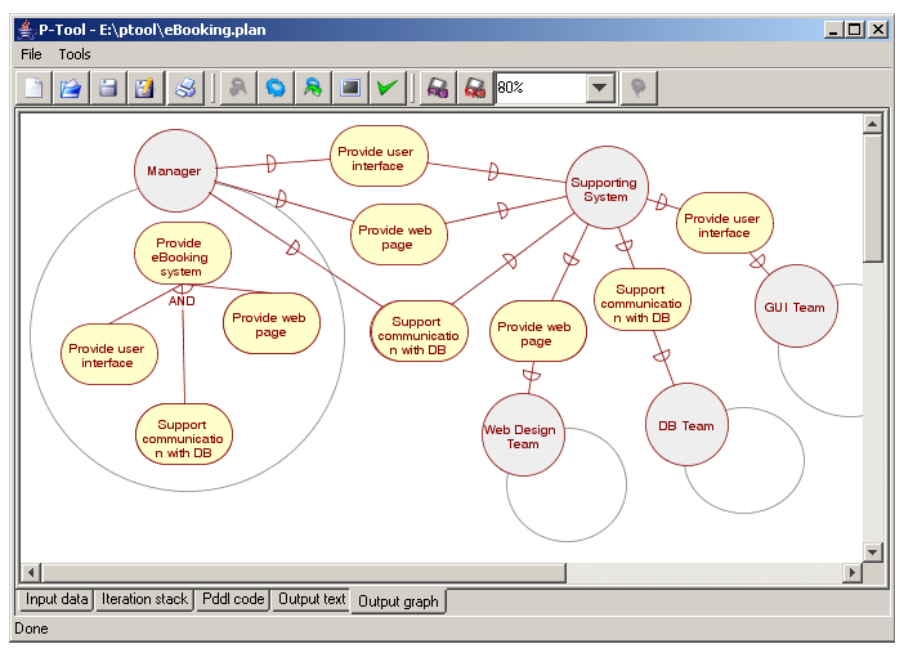

(b) i* diagram for the generated alternative

Fig. 8. P-Tool

with 10 and with 120 "additional" actors is the same (less than one second), only the parsing time increases insignificantly. At the same time, search time for the plan with long delegation chains (more than 30 steps) is much greater (around 15 seconds). Of course, the scalability issue should be explored much more carefully (actually, this is one of our future work plans), but the above reported preliminary experiments have shown promising results. 


\subsection{P-Tool}

We have developed P-Tool, an implemented prototype to support the designer/requirements engineer in the process of exploring and evaluating alternatives. The tool has the interface for the input of actors, goals and their properties, which can be seen in Figure 8a. LPG-td is built in the tool, and is used to generate design alternatives, and represents each solution graphically using $i *$ notation, see Figure $8 \mathrm{~b}$ for an example.

In the following we will illustrate how the steps 3-5 of our approach (see Introduction) could be supported by the P-Tool. For the sake of simplicity we will leave out some details. Steps 1 and 2 are illustrated in Section 3. Predefined upper cost bound $c_{i}^{u p}$ for all actors $a_{i}, i=\overline{1, n}$ is equal to 15 units.

Step 3. First, the planning "on the upper level" for Manager actor is performed. We will skip the process description. The resulting alternative can be seen in the screenshot in Figure 8b. Manager decomposes ProvideEBookingSystem goal into ProvideUI, ProvideWebPage and SupportDBCommunication subgoals, and passes them through the SupportingSystem to GUITeam, WDTeam and DBTeam, respectively.

Step 4. We will illustrate this step with exploring alternatives for the subgoal ProvideWebPage assigned to WDTeam actor. Firstly, an alternative presented in Figure 6 and Figure 7 is generated. For this alternative $c\left(P_{1}, W\right.$ DTeam $)=2+2+4+2+4+2=$ 16 , which does not satisfy WDTeam actor $\left(c_{i}^{u p}=15<16\right)$, so it tries to decrease the imposed load. According to the evaluation procedure described in Section 3.2, the action (SATISFIES WDTeam ProvideRegForm) is selected as $d_{\text {worst }}$. When this action is negated, the planner is not able to find a solution. Thus, the next $d_{\text {worst }}$ is identified, which is (SATISFIES WDTeam DesignFromScratch). New alternative is generated, see Figure 9, for which $c\left(P_{2}\right.$, WDTeam $)=2+2+4+2+2+1=13$. This last alternative is then fixed as it satisfies WDTeam actor.

Step 5. When partial plans are combined into the plan $P$ and evaluated, it appears that $c(P$, GUITeam $)=6$ and $c(P, W D T e a m)=13+6=19$. Actor WDTeam tries to deviate from the alternative $P$, and (SATISFIES WDTeam ProvideBookingWebl) of the plan depicted in Figure 6 is identify as $d_{\text {worst }}$ and negated. By replanning we get an alternative presented in Figure 10, for which $c\left(P^{\prime}\right.$, GUITeam $)=2+2+4+2=10$ and $c\left(P^{\prime}, W D T\right.$ Team $)=13+3=16$. This new alternative satisfies both GUITeam and WDTeam actors.

\section{Related Work}

Modeling requirements and designing information systems and organizations in terms of goals and their interdependences has been a topic of considerable research interest during the last decades [18]. A number of goal-oriented approaches for requirements representation and reasoning were introduced, e.g. KAOS [5]. Requirements engineering is considered to be a crucial part of software development process [18]. Careful elicitation and analysis of requirements help to develop a system that meets user's expectations, is trustful and robust.

The field of AI planning has been intensively developing during the last decades, and has found a number of applications (robotics, process planning, autonomous agents, 


\begin{tabular}{|l|}
\hline (AND_DECOMPOSES WDTeam ProvideWebPage \\
DesignWebPage ProvideRegistrProc) \\
(AND_DECOMPOSES WDTeam ProvideRegForm \\
ConsultOnStoreUData) \\
(SATISFIES WDTeam ProvideRegForm) \\
(PASSES WDTeam DBTeam ConsultOnStoreUData) \\
(SATISFIES DBTeam ConsultOnStoreUData) \\
(OR_DECOMPOSES WDTeam DesignWebPage \\
DesignFromScratch FindStandardTemplate) \\
(PASSES WDTeam SupportingSystem FindStandardTemplate) \\
(SATISFIES SupportingSystem FindStandardTemplate) \\
\hline
\end{tabular}

(a) Plan

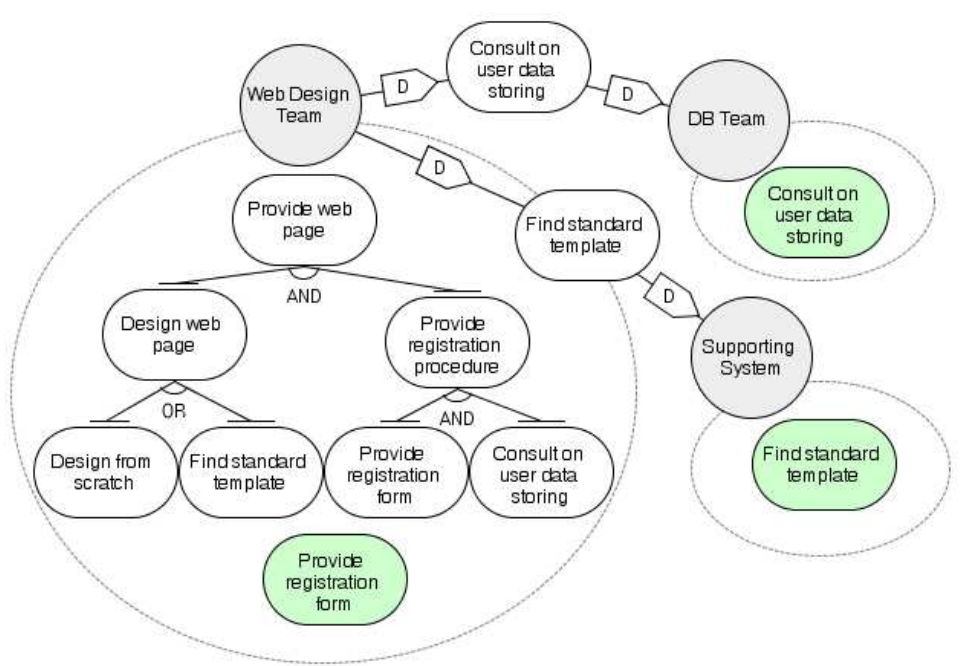

(b) Diagram

Fig. 9. New plan for ProvideWebPage subgoal

etc.). Planning approach recently has proved to be applicable in the field of automatic Web service composition [15]. There are two basic approaches to the solution of planning problems [19]. One is graph-based planning algorithms in which a compact structure, called Planning Graph, is constructed and analyzed. In the other approach the planning problem is transformed into a SAT problem and a SAT solver is used.

There exist several ways to represent the elements of a classical planning problem, i.e. the initial state of the world, the system goal, or the desired state of the world, and the possible actions system actors can perform. The widely used, and to the certain extend standard representation is PDDL (Planning Domain Definition Language), the problem specification language proposed in [10]. Current PDDL version, PDDL 2.2 [6] used during the last International Planning Competition [11], supports many useful features, e.g. derived predicates and timed initial literals. 
\begin{tabular}{l} 
(OR_DECOMPOSES GUITeam ProvideUI ProvideUI1 ProvideUI2) \\
(AND_DECOMPOSES GUITeam ProvideUI2 \\
ProvideBookingGUI ConsultOnGUIToWebAdoption) \\
(SATISFIES GUITeam ProvideBookingGUI) \\
(PASSES GUITeam WDTeam ConsultOnGUIToWebAdoption) \\
(SATISFIES WDTeam ConsultOnGUIToWebAdoption) \\
\hline
\end{tabular}

(a) Plan

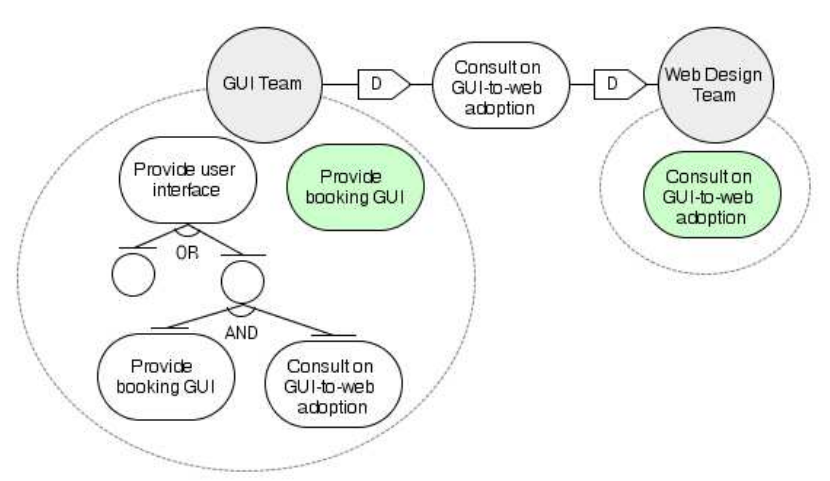

(b) Diagram

Fig. 10. New plan for ProvideUI subgoal

A few works can be found which relate planning techniques with information systems requirements analysis and design. In [1] a program called ASAP (Automated Specifier And Planner) is described, which automates a part of the domain-specific software specification process. ASAP assists the designer in selecting methods for achieving user goals, discovering plans that result in undesirable outcomes, and finding methods for preventing such outcomes. The disadvantage of the approach is that the designer still performs a lot of work manually determining the combination of goals and prohibited situations appropriate for the given application, defining possible start-up conditions and providing many other domain-specific expert knowledge.

Castillo et al. [4] present an AI planning application to assist an expert in designing control programs in the field of Automated Manufacturing. The system they have built integrates POCL, hierarchical and conditional planning techniques (see $[4,15]$ for references). The authors consider standard planning approaches to be not appropriate with no ready-to-use tools for the real world, while in our paper the opposite point of view is advocated. Another recent application of the planning approach to the design of the secure systems is proposed by Gans et al. [9]. The work is based on $i^{*}$ modeling approach [20] and ConGolog (see [15] for description and references), a logic-based planning language. However, the authors focus more on representing/modeling trust in social networks, than on the design automation, and do not go far in explaining how they exploit the planning formalism.

Game theory is an established discipline which deals with conflicts and cooperation among rational independent decision-makers, or players. The key concept in classical 
game theory is the notion of equilibrium [14] which defines the set of strategies, one for each player, which none of the independent rational players wants to deviate from. By playing an equilibrium each player maximizes his utility locally, given some constraints. For example, playing the Nash equilibrium means that no player can benefit when deviating from his equilibrium strategy given that all other players play the equilibrium.

Game theory is applied in various areas, especially in economics (modeling markets, auctions, etc.), corporate decision making, defense strategy, telecommunications networks and many others. Among the examples are the applications of game theory to so called network games (e.g. routing, bandwidth allocation, etc.), see [17] for references.

\section{Conclusions}

We have proposed a framework for automatic exploration the space of alternative actor dependency networks that satisfy an initial set of system actors goals. The framework uses planning techniques to explore the space of design alternatives. A prototype tool with a built-in off-the-shelf planner is used to generate alternatives. These are evaluated in terms of criteria founded on game-theoretic notions.

This is clearly a first step towards making more systematic and tool-supported the process of designing actor dependency models for a given set of initial stakeholder goals. More needs to be done to ensure the scalability of the P-Tool. In particular, we'd like to include the use of heuristic (e.g., $A^{*}$-like [16]) techniques to reduce the space of alternatives under considering by filtering away early on alternatives that look bad. We'd also like to adopt proposals for better structuring actor dependency models. One such proposal [7] is to make $i *$ models "service-oriented" by encapsulating composite actors and allowing delegations to it only through a well-defined service interface. Such proposals reduce dramatically the number of possible solutions to a given multi-actor planning problem.

\section{Acknowledgements}

We thank Alfonso Gerevini and Alessandro Saetti for the support on the use of LPG-td planner. This work has been partially funded by EU Commission, through the SENSORIA and SERENITY projects, by the FIRB program of MIUR under the ASTRO project, and also by the Provincial Authority of Trentino, through the MOSTRO project.

\section{References}

1. J. S. Anderson and S. Fickas. A proposed perspective shift: viewing specification design as a planning problem. In IWSSD '89: 5th Int. workshop on Software specification and design, pages 177-184, 1989.

2. P. Bresciani, P. Giorgini, F. Giunchiglia, J. Mylopoulos, and A. Perini. Tropos: An agentoriented software development methodology. JAAMAS, 8(3):203-236, 2004. 
3. V. Bryl, F. Massacci, J. Mylopoulos, and N. Zannone. Designing secure systems through planning. In CAiSE'06, pages 33-47. Springer, 2006.

4. L. Castillo, J. Fdez-Olivares, and A. Gonzlez. Integrating hierarchical and conditional planning techniques into a software design process for automated manufacturing. In ICAPS 2003, Workshop on Planning under Uncertainty and Incomplete Information, pages 28-39, 2003.

5. A. Dardenne, A. van Lamsweerde, and S. Fickas. Goal-directed requirements acquisition. Science of Computer Programming, 20:3-50, 1993.

6. S. Edelkamp and J. Hoffmann. Pdd12.2: The language for the classical part of the 4th international planning competition. Technical Report 195, University of Freiburg, 2004.

7. H. Estrada. Private communication.

8. M. Fox and D. Long. Pddl2.1: An extension to pddl for expressing temporal planning domains. J. Artif. Intell. Res. (JAIR), 20:61-124, 2003.

9. G. Gans, M. Jarke, S. Kethers, and G. Lakemeyer. Modeling the impact of trust and distrust in agent networks. In AOIS-01, pages 45-58, 2001.

10. M. Ghallab, A. Howe, C. Knoblock, D. McDermott, A. Ram, M. Veloso, D. Weld, and D. Wilkins. PDDL - The Planning Domain Definition Language. In AIPS-98, 1998.

11. IPC-4 Homepage. International Planning Competition 2004. http://1s5-www.cs.unidortmund.de/ edelkamp/ipc-4/.

12. E. Letier and A. van Lamsweerde. Reasoning about partial goal satisfaction for requirements and design engineering. SIGSOFT Softw. Eng. Notes, 29(6):53-62, 2004.

13. LPG Homepage. LPG-td Planner. http://zeus.ing.unibs.it/lpg/.

14. M. J. Osborne and A. Rubinstein. A Course in Game Theory. MIT Press, 1994.

15. J. Peer. Web Service Composition as AI Planning - a Survey. Technical report, University of St. Gallen, 2005.

16. S. Russell and P. Norvig. Artificial Intelligence: A Modern Approach. Prentice Hall, second edition, 2002.

17. E. Tardos. Network games. In Proceedings of the Annual ACM Symposium on Theory of Computing, 2004.

18. A. van Lamsweerde. Requirements engineering in the year 00: a research perspective. In ICSE-00, pages 5-19. ACM, 2000.

19. D. S. Weld. Recent Advances in AI Planning. AI Magazine, 20(2):93-123, 1999.

20. E. S.-K. Yu. Modelling strategic relationships for process reengineering. $\mathrm{PhD}$ thesis, University of Toronto, 1996. 